\title{
Illustrierter Sammelband zur Militärgeschichte
}

FLAVI VEGETI RENATI, VIRI INL.

DE RE MILITARI

LIBRI QVATVOR;

Poft omnes omnium editiones, ope veterum librorum correcti,

GODESCALCO STEWECHIO Humflans.

Accefferunt $S_{E x}$. IVLI FR O N I I NI Strategematôn libri quatuor: E L1 A NVS De inttruendis aciebus: MODes rvs Devocabalis rei militaris: Caftrametatio Romanorum eX hifforiis $\mathrm{P} O \mathrm{~L}$ Y B 1 .

Accesit feorfin eiufdem G. S T E W B C BI in Fl. Vegetium CoMmentaravs.

Adiuncta ciofdem G, Strwe

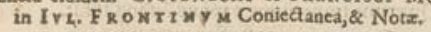

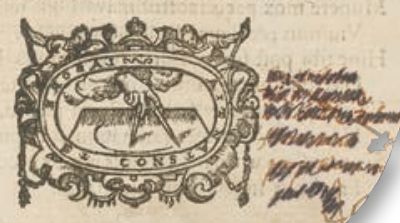

LVGDVNI BATAVORYM,

EX OFFICINA PLANTINIAN

Apud Francifcum Raphelengium. cIo. Io, XCII.

Abb. 1: Titelblatt

374 GODESC. STEVEC. COM.

Tamatis, ${ }^{374}$ Alix dubio procul turres ambulatoria, nec ita fpeciofe,

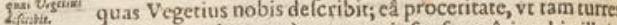
ciuitatis lapideas, guàm muros ipfos fuperârint. his illein inferiori parec ribuit ariecem circa medium verò pontem (cxofrap pofèे vocabic) quem fubirò milites profure-

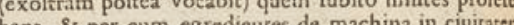
bant, \& per ca vo egr

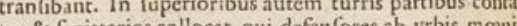

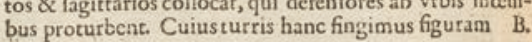

Pons protrufuse ex medio tarris: $C$.

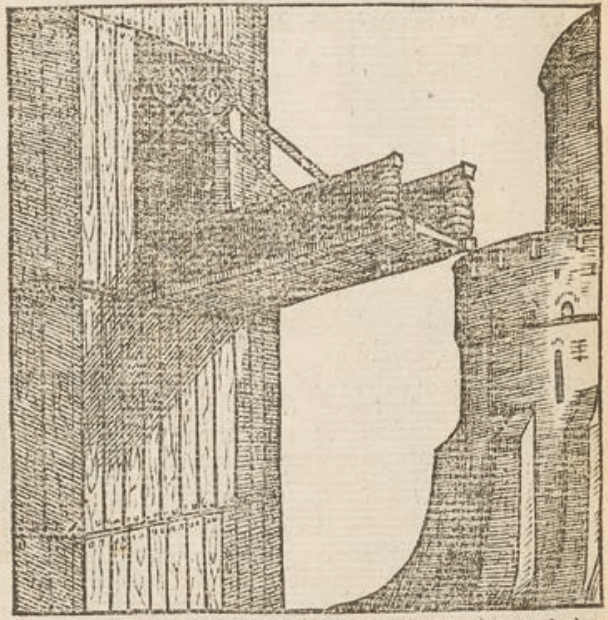

Ponten Vegetius tradit in murri fuiffe, factum de dua bus trabibus, eprem de vimune; quem fubirò prolatum, inter turrem murumçue conflituejant ad egrediendum in

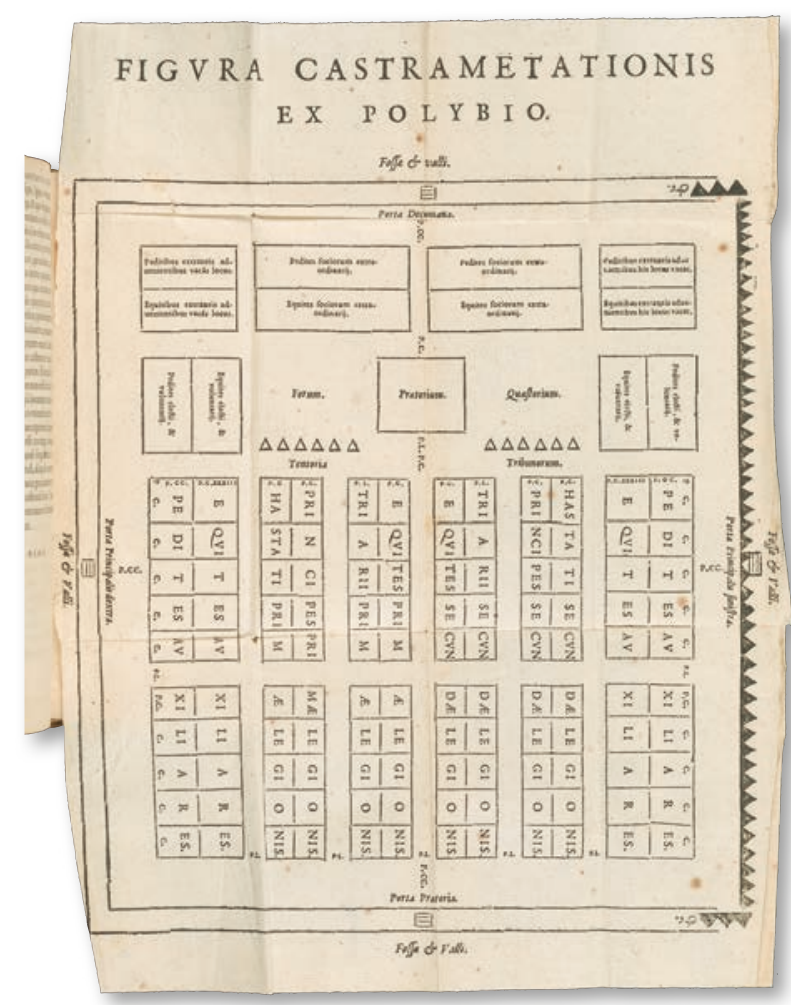

Abb. 2: Falttafel (römisches Heerlager)

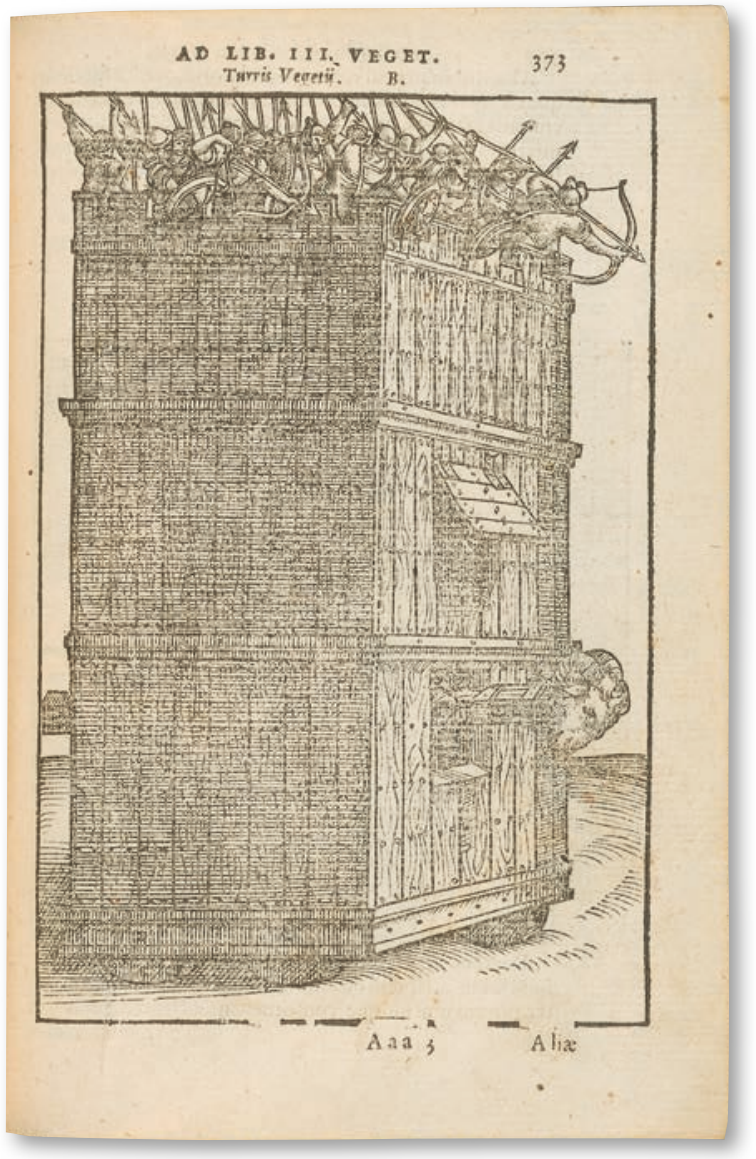

Abb. 3-4: Belagerungsgeräte (Holzschnitte) 
Bei der Stuttgarter Antiquariatsmesse gelang die Erwerbung eines buchgeschichtlich bedeutsamen Sammelbandes (HBF 10265). Zentral ist dabei die Neuedition eines antiken Klassikers zur militärischen Strategie, nämlich der Abhandlung „De re militari" des Flavius Vegetius Renatus (ca. 383450). Dieses Werk weist eine lange Tradition handschriftlicher Überlieferung auf und wurde auch von mehreren Inkunabel-Druckern auf den Buchmarkt gebracht. Die Humanisten interessierten sich aus philologischen, aber auch ethischen Gründen für Vegetius, da seine Ausführungen Fragen der Disziplin und Menschenführung sowie strategischen Planung betrafen. Gerade für Fürsten waren Orientierungspunkte für politisches Handeln enthalten. Dass die WLB auch über eine Augsburger Ausgabe von ca. 1475 in deutscher Übersetzung aus der Hofbibliothek Ottheinrichs von der Pfalz (1502-1559) verfügt (Inc.fol.15916), könnte als Indiz für diese Adressatengruppe gewertet werden.

Die Neuausgabe wurde durch den niederländischen Historiker Godescalcus Stewechius (15511586) vorbereitet und erschien in der Leidener Offizin des humanistischen Gelehrten Franciscus Raphelengius (1539-1597). Raphelengius war der Schwiegersohn des berühmten Antwerpener Buchdruckers Christoph Plantin (1520-1589) und für die Filiale der Plantinschen Buchdruckerei in Leiden verantwortlich. Raphelengius' philologische Kenntnisse trugen neben der technischen
Expertise zu der herausragenden sprachlichen Korrektheit und typographischen Perfektion der Plantin-Drucke bei. Im vorliegenden Sammelband ist außer mehreren kleineren Schriften auch ein ausführlicher Kommentar zu Vegetius enthalten, den Stewechius verfasste. Auch wegen zahlreicher Querverweise auf andere antike Schriften, teils in griechischer Sprache, ist dieser Kommentar wertvoll. Viele Beobachtungen des Vegetius zur Organisation militärischer Einheiten, zum Bau von Kastellen, zu Belagerungstechniken sowie zu Waffen wurden durch Holzschnitte veranschaulicht. Dabei fällt insbesondere bei der Darstellung der Belagerungsgeräte auf, dass die Existenz mittelalterlicher Burgen vorausgesetzt ist, die es zu Vegetius' Zeiten in dieser Form noch nicht gegeben hat. Es handelt sich bei dem Kommentar also auch um eine Aktualisierung. Eine Falttafel stellt hingegen als Illustration zur beigebundenen Schrift "Castrametatio Romanorum“ ein idealtypisches Heerlager der Römer dar, wobei die Heereseinheiten durch von Buchstaben ausgefüllte Vierecke symbolisiert werden.

Christian Herrmann

\section{Pässe litauischer und lettischer Juden: Zeugnisse deutscher Besatzungspolitik im Ersten Weltkrieg}

Im Herbst 2018 erwarb die Bibliothek für Zeitgeschichte (BfZ) zwei Pässe von Juden aus Litauen und Kurland aus den Jahren 1917 und 1918. Die zunächst eher unscheinbar wirkenden Ausweispapiere sind nicht nur sehr selten zu finden, sondern sie erzählen auch eine interessante Geschichte. Die beiden 9x15 cm großen, blassblauen Pässe wurden von der deutschen Verwaltung im Gebiet des Oberbefehlshabers Ost ausgestellt. Der erste Pass gehörte der in Poniewiez (heute Panevėžys) in Litauen wohnhaften Dina Strimling. Für die 1895 geborene Frau ist kein Beruf angeben. Der zweite Pass war Eigentum des 1905 geborenen Israel Melamed. Der Schüler stammte aus der Stadt Tuckum in Kurland, die heute Tukums heißt und zu Lettland gehört. 